EPJ Web of Conferences 66, 07023 (2014)

DOI: $10.1051 /$ epjconf/ 20146607023

(C) Owned by the authors, published by EDP Sciences, 2014

\title{
Relativistic EOS for supernova simulations
}

\author{
H. Shen ${ }^{1, a}$ \\ ${ }^{1}$ School of Physics, Nankai University, Tianjin 300071, China
}

\begin{abstract}
We study the relativistic equation of state (EOS) of dense matter covering a wide range of temperature, proton fraction, and baryon density for the use of supernova simulations. This work is based on the relativistic mean-field theory (RMF) and the Thomas-Fermi approximation. The Thomas-Fermi approximation in combination with assumed nucleon distribution functions and a free energy minimization is adopted to describe the non-uniform matter, which is composed of a lattice of heavy nuclei. We treat the uniform matter and non-uniform matter consistently using the same RMF theory. We compare the EOS tables in detail.
\end{abstract}

\section{Introduction}

The study of dense matter, especially the equation of state (EOS), has been a hot topic in recent years $[1,2]$. The EOS is used as an important input in the studies of neutron stars, supernova simulations, and some other astrophysical processes $[3,4]$. Great efforts have been made by several groups to derive the EOS for supernova simulations and neutron stars [1, 2, 5-7]. However, most of the investigations were restricted to the case of zero temperature and high density with uniform distribution of particles, which were mainly aimed at the study of neutron stars, but not applicable for the use in supernova simulations. At present, there exist two standard EOS's, which are commonly used in supernova simulations, namely the one by Lattimer and Swesty [5] and the one by Shen et al. [6]. The Lattimer-Swesty EOS is based on a compressible liquid-drop model with Skyrme forces. The Shen EOS is based on a relativistic mean-field (RMF) model and uses the Thomas-Fermi approximation in a Wigner-Seitz cell for the description of non-uniform matter. Recently, a Hartree mean-field calculation was performed for the Wigner-Seitz cell in non-uniform matter [7]. The Hartree calculation can incorporate nuclear shell effects, but it requires much more computational resources.

In our previous work [6], we have constructed the EOS table of supernova matter covering a wide range of temperature $T$, proton fraction $Y_{p}$, and baryon mass density $\rho_{B}$ for the use of core-collapse supernova simulations. Recently, the Shen EOS table has been updated with an improved design of ranges and grids according to the requirements of the EOS users [8]. In addition, non-nucleonic degrees of freedom, such as hyperons and deconfined quarks, have been considered at high density by several groups [9-11], and their EOS tables are connected with the Shen EOS at low density.

ae-mail: shennankai@gmail.com 


\section{Method}

We study the EOS for supernova simulations and neutron star calculations in the framework of the relativistic mean-field (RMF) theory. The RMF theory has been successfully employed to study various phenomena in nuclear physics over the past decades [12,13]. The RMF theory was recently reinterpreted by the relativistic Kohn-Sham density functional theory, which has been successfully employed in the treatment of the quantum many-body problem in atomic, molecular, and condensed matter physics. In the RMF approach, baryons interact through the exchange of scalar and vector mesons, and parameters are generally determined by fitting to some nuclear matter properties or ground-state properties of finite nuclei. We calculate the properties of dense matter with both uniform and nonuniform distributions in the RMF framework adopting the TM1 parameter set, which was determined in ref. [14] by fitting some ground-state properties of nuclei including unstable ones. For uniform matter, the RMF theory can be easily used to calculate the properties of matter. For non-uniform matter where heavy nuclei are formed in order to lower the free energy, we use the Thomas-Fermi approximation based on the work by Oyamatsu [15]. The non-uniform matter is modeled as a mixture of a single species of heavy nuclei, alpha particles, and free nucleons that exist outside of nuclei. The results of the RMF model are taken as input in the Thomas-Fermi calculation, so the treatment of non-uniform matter is consistent with that of uniform matter.

For each $T, Y_{p}$, and $\rho_{B}$, we determine the thermodynamically favorable state that has the lowest free energy. We perform the free energy minimization for both non-uniform matter and uniform matter. By comparing the free energies of non-uniform matter and uniform matter, we determine the most favorable state and estimate the phase transition between non-uniform matter and uniform matter.

\section{Results}

We construct the EOS tables covering a wide range of temperature $T$, proton fraction $Y_{p}$, and baryon mass density $\rho_{B}$ for the use of core-collapse supernova simulations. In our earlier work [6], we worked out the relativistic EOS (hereafter referred to as EOS1), which has been widely used in astrophysical studies over the past decade. Recently, we have significantly improved our EOS tables in several aspects according to the requirements of the users of EOS1 [8]. We have presented two sets of EOS tables, namely EOS2 and EOS3, in ref. [8]. EOS2 takes into account only the nucleon degree of freedom, while EOS3 includes additional contributions from $\Lambda$ hyperons. In comparison with the earlier version (EOS1) described in [6], several improvements are made in EOS2 and EOS3. We largely increase the number of $T$ points, and adopt a linear $Y_{p}$ grid in EOS2 and EOS3 instead of the logarithmic $Y_{p}$ grid used in EOS1. On the other hand, the numerical methods are improved to allow the calculation with equal grid spacing for $\rho_{B}$, while it is only approximately equal at high density in EOS1. For a detailed comparison between the EOS tables discussed in this paper, one can see table 1.

It is very interesting to discuss the composition of matter covering the wide range of thermodynamic conditions given in table 1. It is found that heavy nuclei can exist in the medium-density and low-temperature region. The phase of nuclear matter at low density is a homogeneous nucleon gas with a small fraction of alpha-particles, the heavy nuclei are formed at some medium densities where the system can lower the free energy by forming heavy nuclei, and it becomes uniform matter as the density increases beyond $\sim 10^{14.2} \mathrm{~g} \mathrm{~cm}^{-3}$. The starting density of the non-uniform matter phase depends on $T$ strongly, while the ending density is nearly independent of $T$. The non-uniform matter phase can exist only in the low-temperature region $(T<14 \mathrm{MeV})$. It is believed that hyperons can appear around twice normal nuclear matter density [9], and the appearance of hyperons tends to soften the EOS at high density. The first hyperon to appear is $\Lambda$ that is the lightest one with an attractive 
INPC 2013

Table 1. Comparison between the EOS tables discussed in this paper

\begin{tabular}{ccccc}
\hline \hline & & EOS1 & EOS2 & EOS3 \\
\hline Constituents & Uniform matter & $n, p, \alpha$ & $n, p, \alpha, \alpha, \Lambda$ \\
& Non-uniform matter & $n, p, \alpha, A$ & $n, p, \alpha, A$ & $n, p, \alpha, A$ \\
\hline$T$ & Range & $-1.0 \leq \log _{10}(T) \leq 2.0$ & $-1.0 \leq \log _{10}(T) \leq 2.6$ & $-1.0 \leq \log _{10}(T) \leq 2.6$ \\
$(\mathrm{MeV})$ & Grid spacing & $\Delta \log _{10}(T) \simeq 0.1$ & $\Delta \log _{10}(T)=0.04$ & $\Delta \log _{10}(T)=0.04$ \\
& Points & $32(\operatorname{including}(T) 0)$ & $92($ including $T=0)$ & $92($ including $T=0)$ \\
\hline \multirow{3}{*}{$Y_{p}$} & Range & $-2 \leq \log _{10}\left(Y_{p}\right) \leq-0.25$ & $0 \leq Y_{p} \leq 0.65$ & $0 \leq Y_{p} \leq 0.65$ \\
& Grid spacing & $\Delta \log _{10}\left(Y_{p}\right)=0.025$ & $\Delta Y_{p}=0.01$ & $\Delta Y_{p}=0.01$ \\
& Points & $72\left(\operatorname{including}_{p}=0\right)$ & 66 & 66 \\
\hline$\rho_{B}$ & Range & $5.1 \leq \log _{10}\left(\rho_{B}\right) \leq 15.4$ & $5.1 \leq \log _{10}\left(\rho_{B}\right) \leq 16$ & $5.1 \leq \log _{10}\left(\rho_{B}\right) \leq 16$ \\
$\left(\mathrm{~g} \mathrm{~cm}^{-3}\right)$ & Grid spacing & $\Delta \log _{10}\left(\rho_{B}\right) \simeq 0.1$ & $\Delta \log _{10}\left(\rho_{B}\right)=0.1$ & $\Delta \log _{10}\left(\rho_{B}\right)=0.1$ \\
& Points & 104 & 110 & 110 \\
\hline Reference & & {$[6]$} & {$[8]$} & {$[8]$} \\
\hline \hline
\end{tabular}

potential in nuclear matter [9]. For other hyperons, $\Sigma$ and $\Xi$ are now considered to appear at higher densities than $\Lambda$, because $\Sigma$ is suggested to be repulsive and $\Xi$ seems to be weakly attractive in nuclear matter according to recent developments in hypernuclear physics [9]. In order to examine the effect of $\Lambda$ hyperons on the EOS at high density, we take into account the contribution from $\Lambda$ hyperons in EOS3, while other hyperons, such as $\Sigma$ and $\Xi$, are ignored due to their relatively high threshold densities and lack of available experimental data. In figure 1, we show the fraction of heavy nuclei, alpha-particles, protons, neutrons, and $\Lambda$ as a function of $\rho_{B}$ for $Y_{p}=0.3$ at $T=1,10$, and $30 \mathrm{MeV}$. At lower temperatures, such as $T=1 \mathrm{MeV}$ (bottom panel) and $T=10 \mathrm{MeV}$ (middle panel), the matter is a uniform gas of neutrons and protons with a small fraction of alpha-particles at low density. The alpha-particle fraction $X_{\alpha}$ increases with increasing $\rho_{B}$, but the formation of heavy nuclei at low temperatures causes a rapid drop of $X_{\alpha}, X_{p}$, and $X_{n}$, which is due to the fact that heavy nuclei use up most of nucleons in non-uniform matter. When the density increases beyond $\sim 10^{14.2} \mathrm{~g} \mathrm{~cm}^{-3}$, the heavy nuclei dissolve and the matter becomes uniform. The alpha-particles may exist up to $\rho_{B} \sim 10^{14.6} \mathrm{~g} \mathrm{~cm}^{-3}$ where the volume excluded by alpha-particles plays an important role that it is unfavorable to have alpha-particles in the uniform matter at such high density. There is a significant fraction of $\Lambda$ hyperons only at high density above $\rho_{B} \sim 10^{14.6} \mathrm{~g} \mathrm{~cm}^{-3}$. It is shown that $X_{\Lambda}$ increases with increasing $\rho_{B}$, which causes a decrease of $X_{n}$. For higher temperatures, the heavy nuclei cannot be formed, but there are finite values of $X_{\alpha}$, especially at medium densities as shown in the top panel of figure 1 . At $T=30 \mathrm{MeV}$ (top panel), $X_{\Lambda}$ is of the order of $10^{-3}$ at low density, and increases rapidly at high density. We find the contribution from $\Lambda$ hyperons increases with increasing temperature. Since we treat the uniform matter and non-uniform matter consistently using the same RMF theory, all the resulting thermodynamic quantities are consistent and smooth in the whole range [8].

\section{Conclusion}

We have compared several versions of the EOS tables, namely EOS1, EOS2, and EOS3. In comparison with the earlier version EOS1, several improvements have been made in EOS2 and EOS3 according to the requirements of the users. We have largely increased the number of $T$ points and adopted a linear $Y_{p}$ grid in EOS2 and EOS3. We note that EOS2 is an update of EOS1, while EOS3 includes additional contributions from $\Lambda$ hyperons. The EOS tables are available on the Web at http://physics.nankai.edu.cn/grzy/shenhong/EOS/index.html,

http://www.rcnp.osaka-u.ac.jp/ shen/, http://user.numazu-ct.ac.jp/ sumi/eos/index.html. 


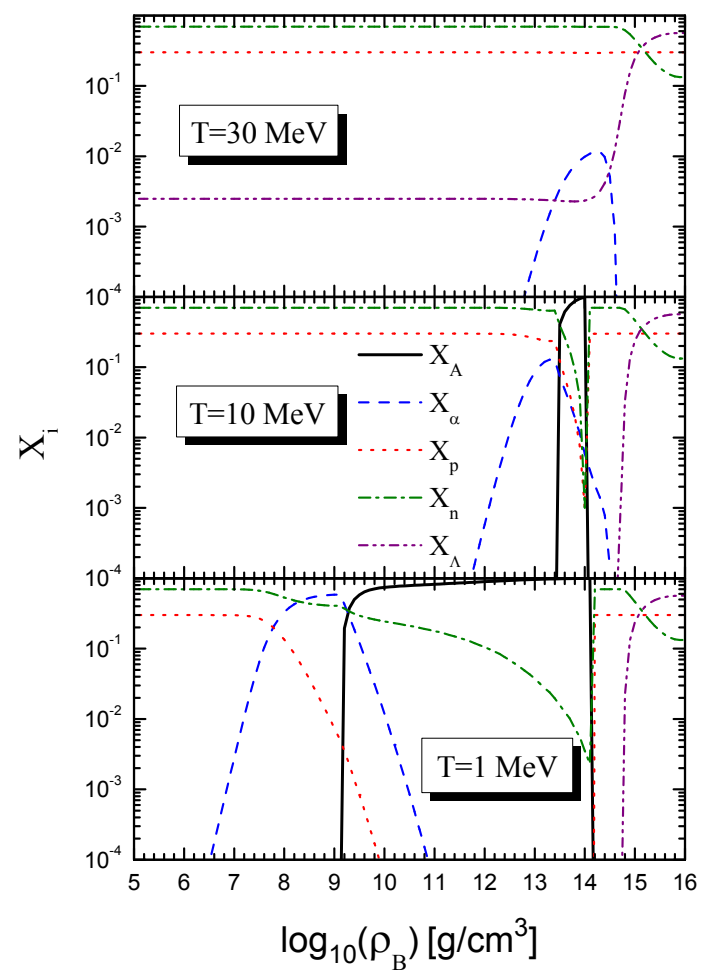

Figure 1. Fraction of heavy nuclei, alpha-particles, protons, neutrons, and $\Lambda$ as a function of the baryon mass density $\rho_{B}$ for $Y_{p}=0.3$ at $T=1,10$, and $30 \mathrm{MeV}$ (bottom to top). The non-uniform matter is modeled as a mixture of free neutrons $(n)$, free protons $(p)$, alpha-particles $(\alpha)$, and a single species of heavy nuclei $(A)$.

\section{References}

[1] F. Weber, Prog. Part. Nucl. Phys. 54, 193 (2005).

[2] J. M. Lattimer, M. Prakash, Phys. Rep. 442, 109 (2007).

[3] H. Th. Janka et al., Phys. Rep. 442, 38 (2007).

[4] K. Sumiyoshi et al., Astrophys. J. 629, 922 (2005).

[5] J. M. Lattimer, F. D. Swesty, Nucl. Phys. A 535, 331 (1991).

[6] H. Shen, H. Toki, K. Oyamatsu, K. Sumiyoshi, Prog. Theor. Phys. 100, 1013 (1998).

[7] G. Shen, C. J. Horowitz, S. Teige, Phys. Rev. C 82, 015806 (2010).

[8] H. Shen, H. Toki, K. Oyamatsu, K. Sumiyoshi, Astrophys. J. Suppl. 197, 20 (2011).

[9] C. Ishizuka, A. Ohnishi, K. Tsubakihara, K. Sumiyoshi, S. Yamada, J. Phys. G 35, 085201 (2008).

[10] K. Nakazato, K. Sumiyoshi, S. Yamada, Phys. Rev. D 77, 103006 (2008).

[11] I. Sagert et al., Phys. Rev. Lett. 102, 081101 (2009).

[12] B. D. Serot, J. D. Walecka, Adv. Nucl. Phys. 16, 1 (1986).

[13] J. Meng et al., Prog. Part. Nucl. Phys. 57, 470 (2006).

[14] Y. Sugahara, H. Toki, Nucl. Phys. A 579, 557 (1994).

[15] K. Oyamatsu, Nucl. Phys. A 561, 431 (1993). 